\title{
Practicable MIMO Capacity in Ideal Channels
}

\author{
S. Amir Mirtaheri,Rodney G. Vaughan \\ School of Engineering Science, Simon Fraser University, British Columbia, V5A 1S6 Canada
}

\begin{abstract}
The impact of communications signal processing such as QAM modulations (instead of gaussian signals), finite block lengths (instead of infinitely long codes), and using simpler algorithms (instead of expensive-to-implement ones), etc., is a lower practicable capacity efficiency than that of the Shannon limit. In this paper, the theoretical and practicable capacity efficiencies for known-channel MIMO are compared for two idealized channels. The motivation is to identify worthwhile trade-offs between capacity reduction and complexity reduction. The channels are the usual complex gaussian random i.i.d., and also the complex gaussian circulant. The comparison reveals new and interesting capacity behaviour, with the circulant channel having a higher capacity efficiency than that of the random i.i.d. channel, for practical SNR values. A circulant channel would also suggest implementation advantages owing to its fixed eigenvectors. Because of the implementation complexity of water filling, the simpler but sub-optimum solution of equal power allocation is investigated and shown to be worthwhile.
\end{abstract}

Keywords- MIMO, capacity, i.i.d., circulant, water-filing, $Q A M$, eigenchannels.

\section{INTRODUCTION}

Multiple-input, multiple-output (MIMO) systems allow increased capacity [1] compared to single port antenna systems in the presence of multipath fading environments. The challenging areas in a MIMO system overlap between the propagation channel, the antennas, and the signal processing [2]. The Shannon, or ergodic (see below), capacity efficiency of MIMO channels has been studied widely, with several accurate expressions available for both the mean and variance. For these results, statistically idealized channels are assumed, and there is an idealized correlation structure from antenna coupling. The basis is information-theoretic with the mutual information being expressed in bits/sec/Hz. The practicable capacity efficiency is for achievable data communications. Its basis is from a combination of information-theoretic and digital communication techniques, and uses the throughput of correct symbols expressed in bits/sec/Hz, but still uses idealized channels. It is of direct interest to engineers designing MIMO systems.

Despite the large body of MIMO literature, there remain aspects which justify further investigation since they can reveal a new appreciation of the capacity-increasing mechanisms, which, in turn, can lead to new implementation strategies.

This paper addresses signal processing aspects for the case of known MIMO channels. This means that, unlike most space-time approaches, there is channel state information at the transmitter. Channel sounding aspects are not addressed here except by reference to the sensitivity of the channel estimates. The channel is therefore considered constant or slow changing.
Known channel techniques do not suit the fast-fading channel where the channel correlation time is within the same order of magnitude as the signaling rate.

The Shannon capacity of the known and unknown channel cases are similar for gaussian channels [2]. However, spacetime codes are about rate half or less. The one exception is the Alamouti code, which is restricted to the smallest MIMO dimension, viz., $2 \times 2$ antenna systems (or sub-systems). For a given capacity efficiency, this is equivalent to a penalty of several $\mathrm{dB}$ of signal-to-noise ratio (SNR). The rate of the spacetime code is therefore an important factor in the capacity efficiency, but having a large MIMO dimension is also important for high capacity efficiency. There are many interpretations of capacity that have arisen in the information theory, signal processing and communications literature. Generally, communications engineers tend to consider capacity in bits/sec, and the capacity efficiency in bits/sec/Hz. Any limiting value refers to Shannons theorem. The more mathematicallybewitched information-theoretic community tends to generate more fundamental results that refer to the gaussian capacity in bits/sec/Hz, and there may be different types of limits. The result is a need for a context-dependent interpretation of "capacity", which can also be "capacity efficiency" and often, both refer to a mean value.

Here, we use the following definition for the theoretical capacity efficiency limit (also known as ergodic capacity, etc.), which we will here call the Shannon capacity,

$$
\frac{C}{B}=E\left\{\sum_{N^{\prime}} \log _{2}\left(1+P_{i} \lambda_{i}\right)\right\} \quad\left(\text { bits } \sec ^{-1} \mathrm{~Hz}^{-1}\right)
$$

$E$ means expectation over the channel realization (which invokes the term 'ergodic'); $N^{\prime}$ is the number of used eigenchannels according to the water filling rule; $\lambda_{i}$ is the $i^{\text {th }}$ eigenvalue of the multipath richness, $\boldsymbol{G}=\boldsymbol{H}^{H} \boldsymbol{H}$ where $\boldsymbol{H}$ is the channel matrix; and $P_{i}$ is the power transmitted to the $i^{\text {th }}$ eigenchannel normalized by the noise at the receiver, so it may be considered as the SNR referred to the transmitter. The gain of the $i^{\text {th }}$ eigenchannel is $\lambda_{i}$ and so $\lambda_{i} P_{i}$ is the SNR at the receiver of the $i^{\text {th }}$ eigenchannel. So for $\lambda>1$ the SNR at the receiver is greater than the SNR at the transmitter.

For the unknown channel case, the equal transmit power allocation is the only logical strategy and space-time coding would be used.

In the known channel case, the estimation of the eigenstructure is critically important. The eigenvectors are needed for the MIMO weights at both ends of the link. Also, the nonlinear power allocation, through water filling, requires 
knowledge of the eigenvalues. This means complex signal processing for dynamic estimation of the eigenvalues and eigenvectors. This paper also looks to situations which lead to a decrease in implementation complexity. If there is a special channel structure, for example the circulant, then there are fixed eigenvectors. This can lead to potential complexity reduction in the signal processing. Another way to reduce the complexity is a sub-optimum power allocation, for example equal power assignment instead of water filling. Examples of the impact on the capacity are presented.

The statistical behaviour of the eigenvalues of a random matrix is complicated [3] and so it is not easy to foresee the effect of channel structure on the MIMO capacity. This unpredictability is worse when the size of the matrix is not very large, e.g. as found in MIMO links. Systematic correlated structures are different to the correlation structure introduced by the antennas and propagation in a wireless MIMO system. Systematic structures include the circulant which has created recent interest [4] [5]. Recent examples of systematic structures are [4] [5] [6] [7] [8]. The emphasis of this paper is on capacity behaviour, and while this includes that of the circulant, it is the comparison itself that leads to a better understanding of MIMO capacity and possible new implementation strategies. Consequently, the practicality of the circulant channel is put aside here, although it is alluded to in [5] and below in the context of wired cables.

\section{CIRCULANT AND RANDOM IID CHANNELS}

The MIMO channel matrix is the usual linear model $\boldsymbol{y}=$ $\boldsymbol{H} \boldsymbol{x}+\boldsymbol{n}$ where $\boldsymbol{x}$ is the input and $\boldsymbol{y}$ is the output and $\boldsymbol{n}$ is noise. An $N \times N$ circulant matrix,

$$
\boldsymbol{H}=\left(\begin{array}{cccc}
h_{0} & h_{1} & \ldots & h_{N-1} \\
h_{N-1} & h_{0} & \ldots & h_{N-2} \\
\vdots & \ddots & \ddots & \vdots \\
h_{1} & h_{2} & \ldots & h_{0}
\end{array}\right)
$$

has known (in terms of the elements) eigenvalues and eigenvectors.

If $\boldsymbol{H}$ is a circulant structure, then $\boldsymbol{G}$ is also circulant. So by having a circulant link, the eigenvectors of $G$ are independent of its eigenvalues, i.e. they are fixed.

Note that a circulant gaussian $N \times N$ link means a link with channel matrix in (2), and a i.i.d. channel means an $\boldsymbol{H}$ with all elements random. The elements of $\boldsymbol{H}$ in both models are zero-mean, unit-variance, complex Gaussian random variables.

It is difficult to arrange a wireless MIMO link (ideally, i.i.d.) to closely approximate a circulant. Nevertheless, in [5], for example, propagation scenarios are offered in an attempt to demonstrate wireless channels which have systematic amplitude correlations such as the real circulant, and an analytic form for the capacity is given. Here, we deal with the complex circulant. It may be possible to arrange a circulant channel structure in a wired MIMO link where there is more access to arranging the physical medium parameters. It is evident that a circularly symmetric arrangement of wires (or wire pairs) [9] could, at least in principle, lead to a circulant channel matrix. Note that because of our choice of known channel capacity definition, the channels do not need to be gaussian distributed.

Theoretical studies of the capacity of MIMO systems show how correlation degrades MIMO capacity under the assumption of certain channel models [10-15]. However, some measurement results show that the capacity degradation might not be as significant as expected [16] and Oestges et. al. [7][8] show a potential benefit of finite correlation.

In wireless MIMO, e.g. [17], a correlated structure exists when correlations among the antennas are finite. So the columns (or rows) of $\boldsymbol{H}$ are independent random vectors, but the elements of each column are correlated with each other and have the same mean and the same covariance matrix. For the case of Rayleigh fading, this implies $E\left\{\boldsymbol{h}_{\boldsymbol{j}}\right\}=\mathbf{0}$ where $\boldsymbol{h}_{\boldsymbol{j}}$ is the $j^{\text {th }}$ column of $\boldsymbol{H}$, and the correlation matrix is $\boldsymbol{\Sigma}=E\left\{\boldsymbol{h}_{\boldsymbol{j}} \boldsymbol{h}_{\boldsymbol{j}}{ }^{H}\right\}$ for $\boldsymbol{j}=1, \ldots, N$. According to this, the circulant matrix in (2) is not a standard correlated MIMO structure because the columns (and the rows) of a circulant $\boldsymbol{H}$ are not independent random vectors. So, with the circulant $\boldsymbol{H}$, we cannot impose a general correlation structure in $\boldsymbol{\Sigma}=E\left\{\boldsymbol{h}_{\boldsymbol{j}} \boldsymbol{h}_{\boldsymbol{j}}{ }^{H}\right\}$ for $\boldsymbol{j}=1, \ldots, N$ as with the i.i.d. channels. Nevertheless, the circulant structures show the same potential beneficial impacts on capacity as the standard correlated channels.

\section{COMPARISON OF THE SHANNON AND THE PRACTICABLE CAPACITIES}

The Shannon capacities for the i.i.d. and circulant random channels can be found from (1). Figure 1 demonstrates the simulation results for the two models. The ordinate is $\sum_{N^{\prime}} P_{i}$ or total SNR. Recall that the $P_{i}$ is the ratio of the transmit power over the noise at the receiver. The receiver noise is assumed here to be equal for all the eigenchannels. In this sense, the ordinate can be considered as the total SNR for the parallel eigenchannels. The MIMO dimension for this example is $20 \times 20$.

For small SNRs, the capacity of the i.i.d. channel is higher than that of the circulant. For large SNRs, it is vice versa. This behaviour is founded on the pdfs of the eigenvalues of the Hermitian matrix of $\boldsymbol{G}$ [4].

For implementation, digital techniques will degrade the capacities from the Shannon limit to the practicable possibilities of a digital link. A starting point is to use the mutual information, $I(\boldsymbol{y} ; \boldsymbol{x})$, for the usual linear model, resulting in the gaussian capacity ([18], [19], [20])

$$
C_{G}(\gamma)=\frac{1}{2} \log _{2}(1+\gamma)
$$

where $\gamma$ is the SNR. The noise pdf is

$$
p_{n}(n)=\frac{1}{\sqrt{2 \pi}} \exp \left(-\frac{1}{2} n^{2}\right) \text {. }
$$




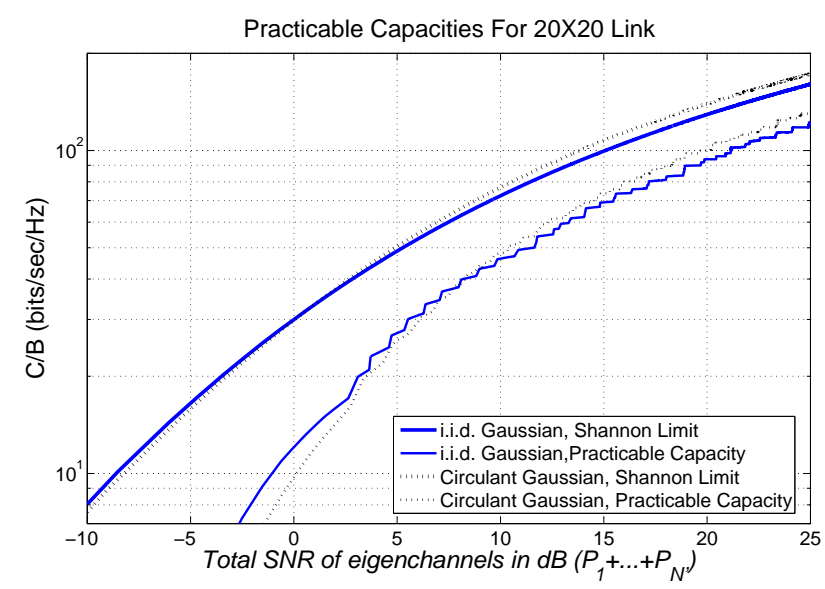

Fig. 1. The Shannon and practicable capacity of the two channel types for $20 \times 20$ systems using QAM and optimum constellation allocation, versus the SNR referred to the transmitter.

So, the output pdf, given the input is

$$
p_{y \mid x}(y \mid x)=p_{n}(n)=p_{n}(y-x) .
$$

The input for $M$-QAM system in xy plane is

$$
\boldsymbol{x}, \boldsymbol{y}=(\ldots,-3,-1,+1,+3, \ldots) \Delta
$$

where the half level spacing, $\Delta$, is determined so that the input variance is $\gamma$, i.e.

$$
\Delta=\sqrt{\frac{3 \gamma}{M^{2}-1}} .
$$

The constellation for $M$-PAM is defined with the input variables. Using (5), $I(\boldsymbol{y} ; \boldsymbol{x})$ for $M^{2}$-QAM is

$$
\frac{2}{M} \sum_{k=0}^{M-1} \int_{-(M-1) \Delta-3}^{(M-1) \Delta+3} p_{n}\left(y-x_{k}\right) \log _{2} \frac{M p_{n}\left(y-x_{k}\right)}{\sum_{i=0}^{M-1} p_{n}\left(y-x_{i}\right)} d y .
$$

The result is shown in Figure 2 plotted against the total QAM SNR $(2 \gamma)$. The digital modulation impose a saturation of capacity. From this saturation level, increasing the power no longer results in a higher mutual information. In practice, for a fading channel, the transmitter should switch the modulation technique to gain capacity [21]. Also, infinitely long data codes are assumed. In a practical situation, data is sent using finite blocks. The impact of the finite block length can be evaluated as follows [18]. For an $M$-QAM, the probability of symbol error could be written as

$$
\operatorname{Prob}_{M}=1-\left(1-P_{\sqrt{M}}\right)^{2} .
$$

where

$$
\operatorname{Prob}_{\sqrt{M}}=\left(1-\frac{1}{\sqrt{M}}\right) \operatorname{erfc}\left(\sqrt{\frac{3 \log _{2} M \gamma_{b}}{2(M-1)}}\right) .
$$

is the probability of symbol error for the $\sqrt{M}$-PAM and $\gamma_{b}$ is the average SNR per bit. To obtain an average bit error rate

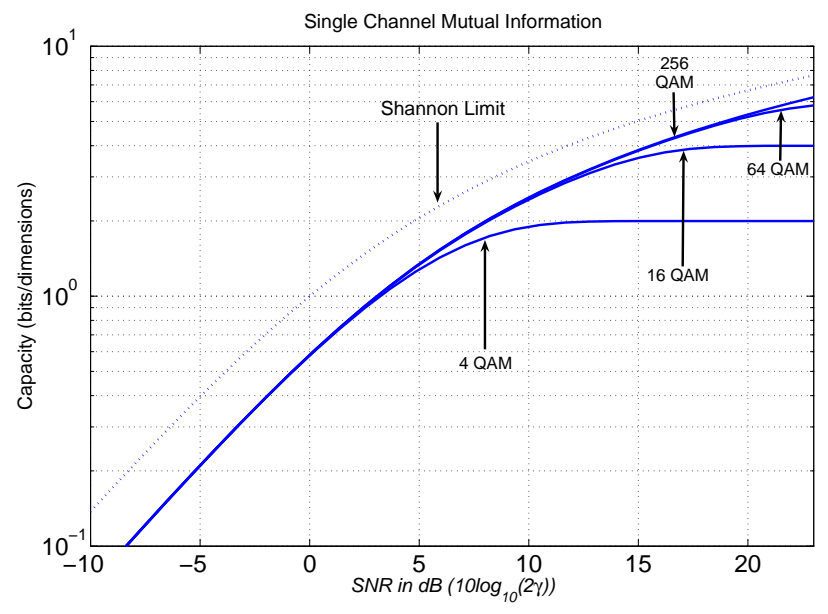

Fig. 2. Single channel capacity penalties in mutual information for QAM versus the SNR referred to the receiver.

(BER), the usual approximation for a probability of bit error is

$$
\operatorname{Prob}_{b} \approx \frac{\operatorname{Prob}_{M}}{\log _{2} M}
$$

To find the capacity from the BERs, a fixed block length of $L$ is assumed. The throughput of only the correctly detected symbols becomes the capacity [22]

$$
\frac{C}{B}=\log _{2} M\left(1-\operatorname{Prob}_{b}(\mathrm{SNR})\right)^{L} .
$$

The block size does not have a large impact for $100<L<$ 10000 , so typically $L=500$ [22]. Figure 3 shows the $7-10 \mathrm{~dB}$

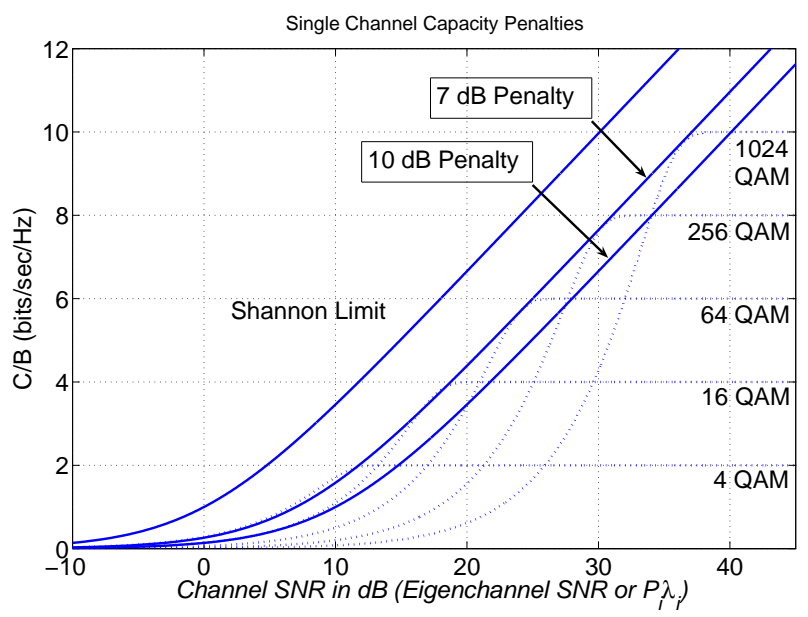

Fig. 3. Single channel capacity penalties for QAM versus the SNR at the receiver.

minimum capacity penalty resulting from using finite block length and uncoded QAM. This penalty can be reduced by the coding gain of forward error correction techniques such as turbo-codes, but this is standard and is not included here. Note the SNR here is referred to the receiver, which allows direct comparison with Figure 2. 
Now, suppose we choose the QAM for each eigenchannel in a standard optimum way so that we remain as close as possible to the Shannon limit, i.e. the most efficient digital technique map for our MIMO system. The table illustrates this QAM selection. Since 1024-QAM is currently very difficult to implement owing to phase noise limitations, we remove it from our consideration. The capacities of the $20 \times 20$ link for the circulant and the i.i.d. cases are shown in Figure 1. Again, for small SNRs, the i.i.d. channel features an advantage while for large SNRs, the circulant structure is better.

\begin{tabular}{|c|c|}
\hline Receiver SNR $\left(P_{i} \lambda_{i}\right)$ & Modulation selection \\
\hline Larger than $27.6(\mathrm{~dB})$ & 256-QAM \\
\hline Between 20.9 and $27.6(\mathrm{~dB})$ & 64-QAM \\
\hline Between 13.2 and $20.9(\mathrm{~dB})$ & 16-QAM \\
\hline Less than $13.2(\mathrm{~dB})$ & 4-QAM \\
\hline
\end{tabular}

TABLE I

THE DIGITAL MODULATION SELECTION STRATEGY

\section{SIGNAL PROCESSING ADVANTAGES OF CIRCULANT STRUCTURES}

MIMO systems are usually very sensitive to errors in the estimation of the eigenvectors. In known channel MIMO theory [2] the Singular Value Decomposition or SVD plays an important role in signal processing. Note that $\boldsymbol{G}=\boldsymbol{H}^{H} \boldsymbol{H}$ is a Hermitian matrix in general and has $N$ distinct, real positive eigenvalues and the remaining will be zero. The SVD expansion of $\boldsymbol{H}$ itself is $\boldsymbol{H}=\boldsymbol{U} \boldsymbol{\Lambda} \boldsymbol{V}^{H}$ where $\boldsymbol{\Lambda}$ is a diagonal matrix of real, non-negative singular values. These singular values are equivalent to the square roots of the eigenvalues of $\boldsymbol{G}$. The columns of the orthogonal matrices $\boldsymbol{U}$ and $\boldsymbol{V}$ are the corresponding singular vectors. Now $\boldsymbol{G}$ can be written as

$$
\boldsymbol{G}=\boldsymbol{H}^{H} \boldsymbol{H}=\boldsymbol{V} \boldsymbol{\Lambda}^{H} \boldsymbol{U}^{H} \boldsymbol{U} \boldsymbol{\Lambda} \boldsymbol{V}^{H}=\boldsymbol{V} \boldsymbol{\Lambda}^{T} \boldsymbol{\Lambda} \boldsymbol{V}^{H},
$$

and $\boldsymbol{V}$ contains the eigenvectors of $\boldsymbol{G}$. Since $\sqrt{\lambda_{i}}$ is the singular value of $\boldsymbol{H}, \boldsymbol{H} \boldsymbol{V}_{i}=\sqrt{\lambda_{i}} \boldsymbol{U}_{i}$ where $\boldsymbol{V}_{i}$ is the $i^{\text {th }}$ column of $\boldsymbol{V}$ and $\boldsymbol{U}_{i}$ is the $i^{\text {th }}$ column of $\boldsymbol{U}$. With $\boldsymbol{V}_{i}$ the transmit weight and $\boldsymbol{U}_{i}^{H}$ the receive weight, the received voltage for this $i^{\text {th }}$ eigenchannel relates to

$$
r_{i}=\boldsymbol{U}_{i}^{H} \boldsymbol{H} \boldsymbol{V}_{i}=\boldsymbol{U}_{i}^{H} \sqrt{\lambda_{i}} \boldsymbol{U}_{i}=\boldsymbol{U}_{i}^{H} \boldsymbol{U}_{i} \sqrt{\lambda_{i}}=\sqrt{\lambda_{i}} .
$$

Now consider we have error in the estimation of the eigenvectors at the transmitter. Denote $\widehat{V}=\boldsymbol{V}+\boldsymbol{n}$ where $\boldsymbol{n}$ is the estimation noise. Then instead of $\boldsymbol{\Lambda}=\boldsymbol{U}^{H} \boldsymbol{H} \boldsymbol{V}$ we will have $\widehat{\Lambda}=\boldsymbol{U}^{H} \boldsymbol{H} \widehat{\boldsymbol{V}}$ in which $\widehat{\boldsymbol{\Lambda}}$ is no longer diagonal.There is now interference (power leakage) between the imperfect eigenchannels and this can severely degrade the MIMO performance. In the case of the circulant channel, the fixed eigenvectors can be exploited to ease this sensitivity. Specifically, the noisy estimation of each of the eigenvectors, $\widehat{\boldsymbol{V}}_{i}$, is compared to the fixed eigenvectors and closest fixed eigenvector is chosen for the transmitter weights. Simulations show that the performance of the noisy circulant MIMO systems can improve considerably whereas it is not possible to recover the i.i.d. capacity using this technique.

\section{SHANNON AND PRACTICABLE CAPACITIES USING EQUAL POWERS}

The ideal transmitting strategy is water filling [23, theorem 7.5.1]. The water filling requires a communication protocol to set up the eigenchannels. This section investigates how much degradation occurs by simply omitting water filling. The total power allocated by the water filling is now equally divided between the eigenchannels. Figure 4 shows the results for the case of the circulant channel and the two power allocation strategies. It is clear that for large SNRs the degradation arising from equal power allocation is negligible. This is a significant but reasonable result since for large SNRs the difference between the water filling powers assigned to different eigenchannels reduces, and so the capacity converges to that of equal power allocation. The result for the i.i.d. channel is very similar with the slight difference that the convergence is faster for the circulant case, owing to the more similar eigenchannels. It is apparent that these two methods of power allocation for the $N \times N$ link converge later, i.e. for larger SNRs, when $N$ is larger. Figure 5 illustrates the practicable

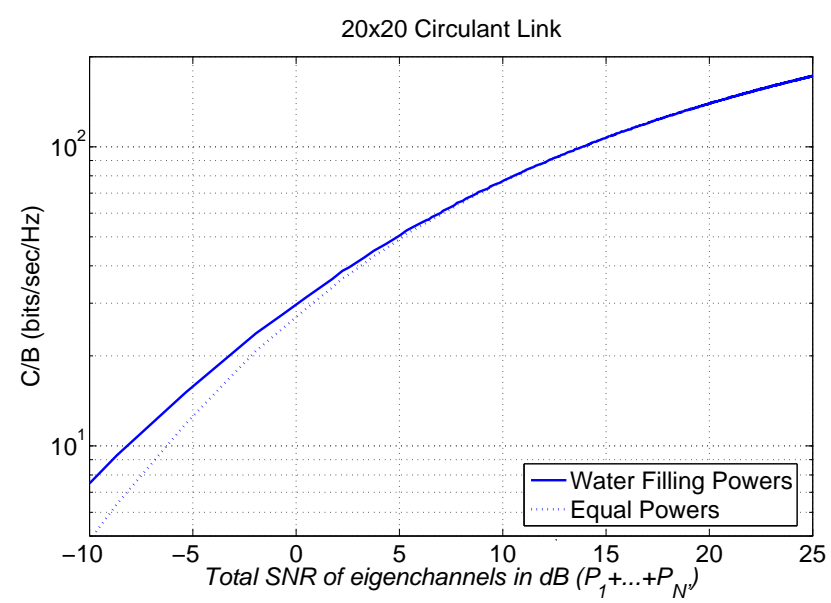

Fig. 4. The Shannon capacity of equal powers and optimum water filling powers for the circulant link

capacities. The advantage of the circulant channel, without the complexities of water filling is clear especially for the SNR. Again the converging capacities of the two methods of power allocation is obvious. This convergence is faster for the circulant case compared to the i.i.d. case. Finally, for this $20 \times 20$ case, in the (total) SNR range over $5 \mathrm{~dB}$, it would be reasonable to use equal powers instead of water filling powers. The practical capacity of the strongest eigenchannel is also depicted. To plot these single eigenchannel capacities in Figure 5, the total amount of power distributed between the different eigenchannels in the MIMO case is assigned to the strongest eigenchannel. For small SNR, putting all of the power in the strongest channel is better than distributing it between many channels, while for large SNRs, both the Shannon and practicable MIMO capacity are much more than the best that could be achieved by a single channel. 


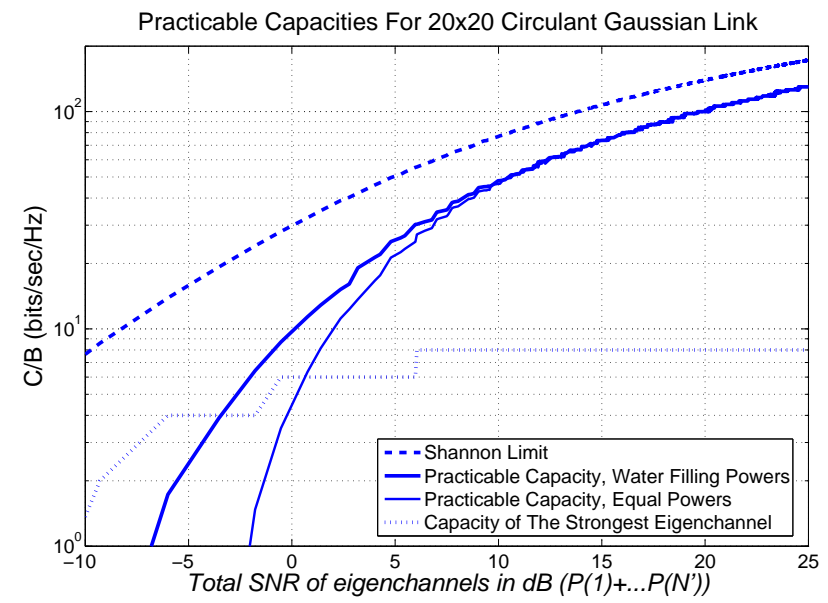

Fig. 5. The practicable capacity of equal powers and optimum water filling powers for the circulant random link using QAM techniques

\section{CONCLUSION}

The practicable capacity efficiency offers a MIMO performance limit which accounts for the use of signal processing. This specifically includes digital modulations and finite block coding, and in the case of known channel MIMO, water filling. The known channel case has been treated here, although a similar capacity efficiency can be expected from the unknown channel case if the space-time coding could be of rate one or very close to rate one. The practicable capacity efficiency is also an achievable capacity of course, as long as the signal processing is correctly implemented and the channels follow the assumed models. The inclusion of the circulant as a channel model is revealing. Despite its structure being a correlated form, the circulant has higher Shannon and practicable capacities than those for the i.i.d. channel. This is because the circulant has more similar eigenvalues. The optimum practicable capacity efficiency follows the trend of the Shannon capacity efficiency, the difference being almost recoverable through the use of finite block length forward error correction coding. The water filling process, associated with known channel MIMO, is expensive on processing power. The simplified strategy of using equal powers for the eigenchannels does not significantly degrade the practicable capacity - for example for SNRs over $5 \mathrm{~dB}$ in a $20 \times 20$ system there is a negligible degradation.

\section{REFERENCES}

[1] J. H. Winters, On the capacity of radio communication system with diversity in Rayleigh fading environment, Commun., vol.JSAC-5, pp.871-878, June 1987.

[2] R. G. Vaughan and J. B. Andersen, Channels, Propagation and Antennas for Mobile Communications, IEE Peregrinus, 2003.

[3] A. T. James, Distribution of matrix variates and latent roots derived from normal samples, Ann. Math. Statist., Vol. 35, pp. 475-501.
[4] S. A. Mirtaheri and R. Vaughan Circulant Wired MIMO Structures, 2005 IEEE Canadian Conference on Electrical and Computer Engineering, May 2005.

[5] H. Ozcelik and C. Oestges Capacity of Diagonally Correlated MIMO Channels, IEEE VTC, May-June 2005.

[6] H. Ozcelik and C. Oestges Some Remarkable Properties of Diagonally Correlated MIMO Channels, electronic Letters, submitted.

[7] C. Oestges, B. Clerckx, D. Vanhoenacker-Janvier, and A. J. Paulraj, Impact of diagonal correlations on MIMO capacity: application to geometrical scattering models, Veh. Tech. Conf. '03, vol. 1, pp. 394-398, Oct. 2003.

[8] C. Oestges, and A. J. Paulraj, Beneficial impact of channel correlations on MIMO capacity, Electronics Lett., vol. 40, Issue 10, pp. 606-608, 13 May 2004.

[9] T. Magesacher, W. Henkel, G. Taubock, and T. Nordstrom, Cable measurements supporting xDSL technologies, Journal e\&i Elektrotechnik und Informationstechnik, 199(2):37-43, Feb. 2002.

[10] A. Paulraj, R. Nabar, and D. Gore, Introduction to space-time wireless communications, Cambridge University Press, Cambridge, UK, 2003.

[11] M. Kang, and M. S. Alouini, Impact of correlation on the capacity of MIMO channels, Proc. IEEE ICC '03, Anchorage, AK, USA, pp. 2623-2627, 2003

[12] V. Raghavan, and A. M. Sayeed, MIMO capacity scaling and saturation in correlated environments, Proc. IEEE ICC '03, Anchorage, AK, USA, pp. 3006-3010, 2003.

[13] C. N. Chuah, D. N. C. Tse, J. M. Kahn, and R. A. Valenzuela, Capacity scaling in MIMO wireless systems under correlated fading, IEEE Trans. Inform. Theory, vol. 48, pp. 637-650, July 2002.

[14] P. J. Smith, L. M. Garth, and S. Loyka, Exact capacity distributions for MIMO systems with small number of antennas, IEEE Commun. Lett., vol. 7, pp. 481-483, 2003.

[15] C. S. Withers, and R. G. Vaughan, Distribution and percentiles of channel capacity for multiple arrays, Proc. 11th Virginia Tech/MPRG Symposium on Wireless Communications, PP. 141-152, June 2001.

[16] C. C. Martin, J. H. Winters, and N. R. Sollenberger, Multiple-input multiple-output (MIMO) radio channel measurements, Proc. IEEE Veh. Tech. Conf. 2000, Boston, MA, USA, pp. 774-779, 2000.

[17] M. Chiani, M. Z. Win, and A. Zanella, On the capacity of spatially correlated MIMO Rayleigh-fading channels, IEEE Trans. Inform. Theory, vol. 49 , Issue 10, pp. 2363-5371, Oct. 2003.

[18] J. G. Proakis, Digital Communications, Fourth ed. McGrawHill, 2000.

[19] T. M. Cover, and J. A. Thomas, Elements of Information Theory, John Wiley \& Sons, 1991.

[20] J. M. Wozencraft, and I. M. Jacobs, Principles of Communication Engineering, John Wiley \& Sons, 1965.

[21] A. .J. Goldsmith and S. G. Chua, Adaptive coded modulation for fading channels, IEEE Trans. Commun., vol. 46 , Issue 5, pp. 595-602, May 1998.

[22] S. Catreux, P. F. Driessen and L. J. Greenstein, Data throughputs using multiple-input multiple-output (MIMO) techniques in a noise-limited cellular environment, IEEE Trans. Wireless Commun., vol. 1, Issue 2, pp. 226-235, April 2002.

[23] R. Gallager, Information theory and reliable communication, John Wiley \& Sons, New York, 1968. 